\title{
Thaumalea angelieri n. sp., Diptère Thaumaleidae nouveau des Pyrénées
}

\author{
F. Vaillant 1 \\ G. Vinçon ${ }^{2}$
}

\begin{abstract}
Mots clés: Diptera, Thaumaleidae, nouvelle espece, écologie, Pyrénées.
Une nouvelle espèce de Diptères Thaumaleidae est découverte dans les Pyrénées et vient s'ajouter aux six autres connues de cet ensemble montagneux; elle est proche d'une espèce du centre de l'Espagne.
\end{abstract}

Thaumalea angelieri n. sp., a new Thaumaleidae (Diptera) from the Pyrenees.

Keywords : Diptera, Thaumaleidae, new species, ecology, Pyrenees.

A new species of Thaumaleidae (Diptera) has been discovered in the Pyrenees ; it is the seventh one known from this mountainous region; it is close to another species from the central part of Spain.

Actuellement 7 espèces de Thaumaleidac seulement sont connues des Pyrénées (Vaillant 1968, 1977), dont 4, Thaumalea austriaca Edwards, Th. miki Edwards, Th. testacea (Ruthé) et Th. verralli Edwards, ont une vaste répartition en Europe. Les 3 autres semblent ètre endémiques des Pyrénées; ce sont Th. pachystyla Vaillant, Th. thomasi Vaillant et $T h$. pyrenaica Edwards; cette dernière espèce, très particulière et sans relations apparentes avec d'autres, est extrêmement abondante dans le bassin de la Neste d'Aure. Les Thaumaleidae ont été peu recherchés dans les Pyrénées et plusieurs autres espèces doivent certainement s'y trouver. En effet les Alpes dans leur ensemble comptent 27 espèces, dont 18 sont endémiques.

L'un de nous a découvert des représentants d'une espèce nouvelle, dont nous décrirons l'imago or et l'imago $९$. Précisons que des individus des deux

1. Laboratoire de Zoologie, Université scientifique et médicale de Grenoble, 38402 Saint-Martin-d'Heres Cédex.

2. Laboratoire d'Hydrobiologie. UA 695 du C.N.R.S.. 118, route de Narbonne, 31062 Toulouse Cédex. sexes ont été trouvés le même jour au même endroit et que les mâles recueillis appartenaient tous à la même espèce ; l'appartenance à celle-ci des femelles, toutes semblables les unes aux autres, n'est assurément pas certaine, car des couples formés n'ont pas été observés. Précisons également que la nomenclature des genitalia $\sigma$ est celle de Vaillant (1982) et que celle des genitalia $\&$ est empruntée à Saether (1977).

\section{Thaumalea angeliert n.sp.}

IMAGO $\sigma$ :

Tergite abdominal IX 1,2 fois plus large que long et peu rétréci dans sa partie distale (fig. 1) ; bord du tergite sans saillie ni échancrure, sauf celle, très peu profonde, qui se trouve au niveau des cerques. Bande sternale IX grêle, cependant un peu épaissie dans sa partie médiane. Plaque post-sternale aussi longue que les gonocoxites et s'amincissant à son extrémité. Gonocoxites renflés et moins de 2 fois plus longs que larges en vue ventrale; gonostyles épais dans leur $1 / 2$ proximale, puis brusquement 


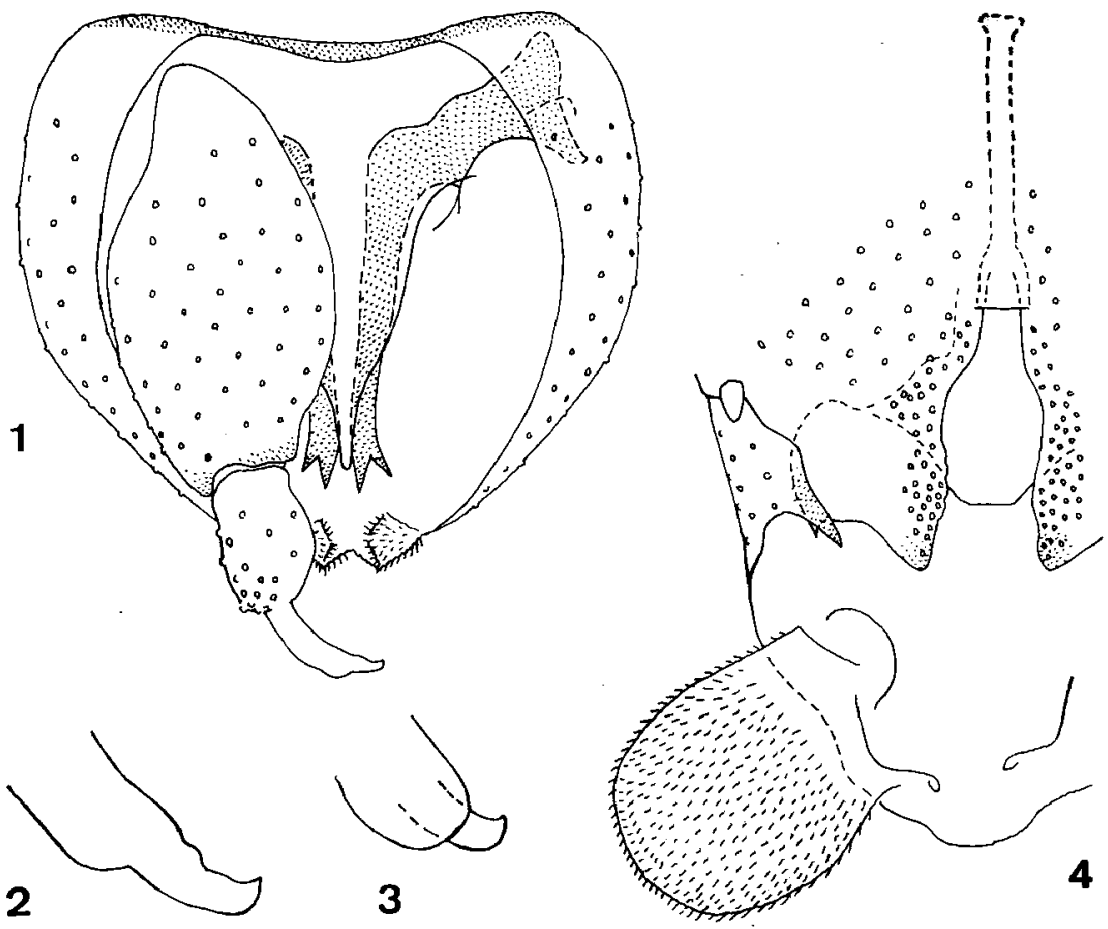

Fig. 1 - 4 : Thaumalea angeliert $n$. sp.

1-3: imago $\sigma-1$ : genitalia, face ventrale -2 et 3 : partie distale d'un gonostyle droit, détail 4 : imago $\&$, genitalia, face ventrale. 


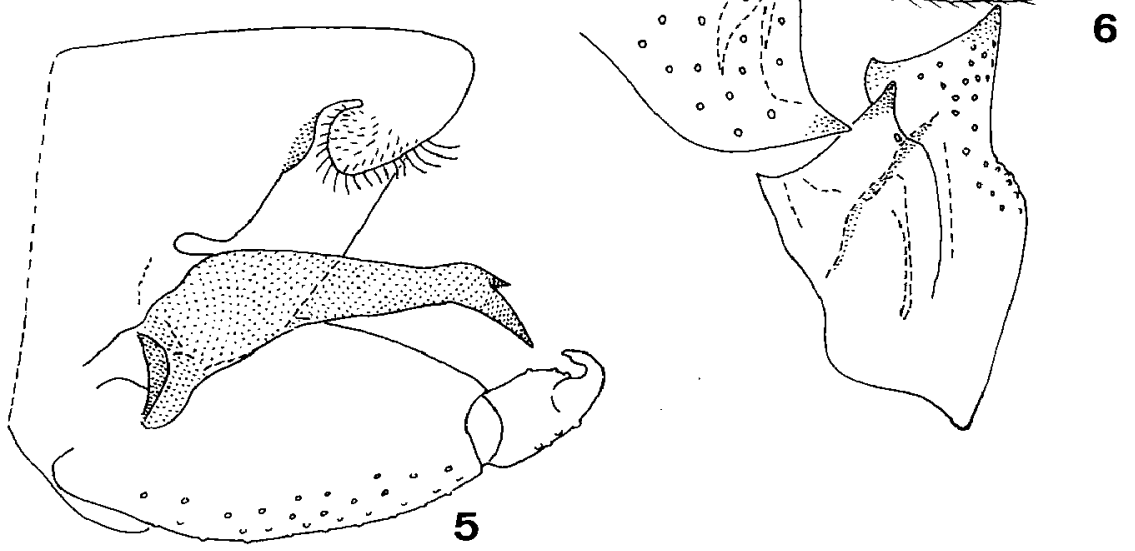

Fig. 5 et 6 : Thaumalea angelieri $\mathrm{n}$. sp.

5: imago $\sigma^{\circ}$, moitié droite du segment génital, profil -6 : imago $९$, genitalia, profil. 
atténués et ensuite de largeur constante ; chaque gonostyle a un bec terminal, déprimé latéralement et de forme assez variable (fig. 2 et 3 ), et n'a pas de denticules. Epiparamères épais en vue latérale (fig. 5) et grèles en vue ventrale (fig. 1); chaque épiparamère $a$, aux $5 / 6$ de sa longueur, une petite épine dirigée dorsalement.

Longueur de l'aile : $3,8 \cdot 3,9 \mathrm{~mm}$.

IMAGO $\&$ :

Gonapophyses VIII courtes, cependant acuminées à leur apex. Le sternite abdominal VIII a des saillies latérales pointues, mais courtes (fig. 4 et 6 ). Il $\mathrm{y}$ a des gonocoxites IX longs et aigus, comme pour Th. freyi Edwards. Le notum est épais et élargi à son extrémité antérieure. Il n'y a pas de branches dorsales de la gonapophyse IX.

L'holotype, un autre $\sigma$ et $4 \&$ ont été pris le 31.IX.1984 à $1600 \mathrm{~m}$ d'altitude en bordure du gave de Bious, qui est un tributaire du gave d'Ossau, dans les Pyrénées Atlantiques.

Nous dédions avec plaisir cette espèce à Monsieur E. Angelier, Professeur à l'Université de Toulouse.

Th. angelieri est proche de Th. gredosensis Schmid (1951), dont le type a été découvert dans la Sierra de Gredos, laquelle est située dans la partie centrale de l'Espagne. L'imago $\sigma$ de cette dernière espèce a été redécrite en 1977 par Vaillant, mais l'imago $\varnothing$ est encore inconnue. Nous ne comparerons donc que les imagos $\sigma^{*}$ des deux espèces voisines. Th. gredosensis se sépare de $T h$. angelieri par les caractères suivants : la bande sternale IX est étroite sur toute sa longueur. La plaque poststernale est large et beaucoup plus courte que les épiparamères. Les gonocoxites sont grêles eł près de 3 fois plus longs que larges en vue ventrale. Les gonostyles sont progressivement atténués vers leur extrémité et ont un denticule terminal partant d'un anneau basilaire. Enfin Th. gredosensis est beaucoup plus petite que Th. angelieri.
Th. gredosensis et Th. angelieri font partie du groupe III a (Vaillant 1969) parmi les Thaumalea. La femelle de $T h$. angelieri rappelle un peu celle de Th. major Bezzi, qui appartient à ce groupe (Vaillant 1981), mais pas du tout celle de Th. miki Edwards, qui en fait partie également (Martinovsky \& Rozkosny, 1976). Par contre, elle se rapproche beaucoup de la femelle de Th. freyi Edwards; or, cette dernière espèce ne fait pas partie du groups III a et le $\sigma$ est très différent de celui de $T h$. angelieri. Il ne faut sans doute pas s'attendre à ce qu'une parenté de caractères des genitalia chez un sexe entraine une parenté comparable chez l'autre sexe parmi les Thaumaleidae.

\section{Travaux cltés}

Martinovsky (J.) \& Rozkosny (R.). 1976. - Four new species of Thaumaleidae (Diptera) from Europe with taxonomic and distributional notes on other species. Acta ent. bohemoslov., 73 (3) : 187-205.

Saether (A.O.), 1977. - Female genitalia in Chi ronomidae and other Nematocera. Bull. Fisher. Res. Bd. Canada, Otawa, $197:$ 1-209.

Schmid (F.). 1951. - Notes sur quelques Thaumaleides suisses et espagnols. Bull. Inst, roy. Sci. nat. Belgique, 29.

Vaillant (F.). 1968, - Les Diptères Thaumaleidae des Pyrénéc' Annis Limnol., 4 (1) : 81-84.

Vaillant (F.). 1969. - Les Diptères Thaumaleidae des Alpes et des Carpathes, Ann. Soc. ent. Fr. (N.S.), 5 (3) : $687-705$.

Vaillant (F.). 1977. - Les Diptères Thaumaleidae d'Europe. Arn. Soc. ent. Fr. (N.S.) 13 (4) : 695-710.

Vaillant (F.). 1981. - Some Diptera Thaumaleidae from Europ Aquatic Insects, 3 : 129-146.

Vaillant ( $F$.). 1982. - Homologies entre les pièces génitales máles de quelques Diptères Nématocères. Ann. Soc. ent. Fr. (N.S.), 18 (3) : $419-425$. 